
allemande

\title{
1917, année de rupture : le rôle du pain de guerre (Kriegsbrot) en Allemagne
}

\section{Nina Régis}

\section{(2) OpenEdition \\ 1 Journals}

Édition électronique

URL : https://journals.openedition.org/allemagne/584

DOI : 10.4000/allemagne.584

ISSN : 2605-7913

Éditeur

Société d'études allemandes

Édition imprimée

Date de publication : 29 décembre 2017

Pagination : 407-422

ISSN : 0035-0974

\section{Référence électronique}

Nina Régis, « 1917, année de rupture : le rôle du pain de guerre (Kriegsbrot) en Allemagne », Revue d'Allemagne et des pays de langue allemande [En ligne], 49-2 | 2017, mis en ligne le 29 décembre 2018, consulté le 18 mai 2021. URL : http://journals.openedition.org/allemagne/584 ; DOI : https://doi.org/ 10.4000/allemagne.584 


\section{7, année de rupture: le rôle du pain de guerre (Kriegsbrot) en Allemagne}

\section{- Nina Régis*}

Nous connaissons les grandes césures militaires de la Grande Guerre. Au niveau mondial, l'année 1917 demeure, avec l'entrée en guerre des États-Unis, un tournant incontesté. Mais connaissons-nous les ruptures du temps de guerre à «l'arrière»? Pour l'Allemagne, cette question importe d'autant plus que l'espace de l'arrière s'étendait à tout le pays, les lignes de front se situant à l'extérieur des frontières. Événements ponctuels d'une part et perceptions des acteurs d'autre part nous permettront d'argumenter en faveur d'une "année de rupture» également à l'arrière, en Allemagne, en 1917. En France en effet, l'arrière dut certes endurer bon nombre de privations et de restrictions matérielles contraignantes. Mais en Allemagne, entre 1914 et 1919 et surtout lors de «l'hiver des navets» en 1916-17, la sous-nutrition mena à une augmentation spectaculaire des maladies et des décès. Dans ce contexte, notre étude sera menée à travers le prisme d'un aliment de base dont les quantités disponibles et l'évolution de la qualité furent décisives: le pain. À travers ses utilisations symboliques, il sera possible d'aller au-delà de l'objet matériel et d'examiner le rôle attribué à la nourriture au cours d'une guerre totale par les civils et les autorités de l'arrière. On pourra ainsi avoir accès à un phénomène décrit par Jean-Jacques Courtine et Georges Vigarello, celui de l'épuisement des corps à travers les restrictions alimentaires, phénomène qui transforme les civils en cibles privilégiées des conflits ${ }^{(1)}$. Grâce à des correspondances, des documents administratifs issus des archives nationales de Berlin (Bundesarchiv Lichterfelde, BArch en note) et des journaux intimes conservés dans le fonds d'archives d'Emmendingen dans le Bade-Wurtemberg (Tagebucharchiv, TArch en note), nous montrerons en quoi non seulement les restrictions alimentaires, mais aussi les

* Doctorante contractuelle avec avenant d'enseignement à l'Université Toulouse-Jean Jaurès, Centre de recherches et d'études germaniques (CREG).

1 Cf. Jean-Jacques Courtine, Georges Vigarello et Alain Corbin (dir.), Histoire du corps, t. 3, Paris, Seuil, 2005, p. 283. 
représentations du pain menèrent au paroxysme de ce phénomène touchant les corps en 1917, année qui représente un moment clé dans un combat silencieux, concernant la sphère de l'intime. 1917, c'est aussi l'année qui, plus que toutes les autres, regorge de sources sur les représentations du pain, intrinsèquement liées à l'expérience de la faim. Enfin, nous montrerons en quoi l'année 1917 fut tout particulièrement le théâtre d'une «vaste expérience naturelle», d'une «immense expérience de psychologie sociale, d'une richesse inouïe » ${ }^{(2)}$ pour reprendre les termes de Marc Bloch en 1921.

\section{Un contexte difficile en début d'année}

Parmi les conditions qui firent de 1917 l'année de guerre la plus rude pour l'arrière, comptent évidemment le manque de main-d'œuvre, le blocus, l'hiver, mais aussi les espoirs et les déceptions liées aux mesures gouvernementales. Par ailleurs, les ruptures de cette année 1917 ont été vécues très différemment selon les acteurs.

\section{A. Rationnement et ersatz: le moral au détriment de la santé?}

Contrairement au reste de la population, les hommes politiques allemands étaient conscients que l'Allemagne entrait dans la guerre en étant dépendante des importations de l'étranger pour un tiers des céréales consommées ${ }^{(3)}$. Pourtant, le programme Hindenburg misait sur la production d'armes, de munitions, de charbon et de fer, non sur l'agriculture. Menant à une restructuration trop hâtive, il fut aussi en partie responsable de l'effondrement de l'économie allemande et, à plus long terme, d'une diminution drastique de la production agraire ${ }^{(4)}$. Dans ce contexte, la politique du pain, pourtant encore peu connue aujourd'hui, eut un impact majeur sur la santé publique. Et avec la mise en place d'un blocus maritime dès le début de la guerre, le pain fut très rapidement dans la ligne de mire des autorités militaires ayant de facto pris le pouvoir décisionnel en 1914.

La première mesure prise fut la mise en place, dès le $1^{\text {er }}$ décembre 1914 , du pain de guerre, Kriegsbrot ou K-Brot. Ce pain devait contenir au moins $5 \%$ de pommes de terre et être marqué d'un "K". Au-delà de $20 \%$, le pourcentage de pommes de terre devait être spécifié ${ }^{(5)}$. Le rationnement s'appliqua d'abord au pain et à la farine ${ }^{(6)}$. Le rationnement du pain fut, grâce à un système de tickets, mis en place à Berlin dès le

2 Marc Bloch, «Réflexions d'un historien sur les fausses nouvelles de la guerre», Bulletin de la Société des Professeurs d'Histoire, t. XXXIII (1921), p. 19.

3 Dès 1915, le ministre de la Guerre de Bavière exprime ses craintes d'une famine dans le cas d'une prolongation des hostilités dans un rapport «très secret» adressé à différents ministres. Voir Erich Matthias, Hans-Meier Welcker (dir.), Quellen zur Geschichte des Parlamentarismus, zweite Reihe Militär und Politik, vol. 1 : Militär und Innenpolitik im Ersten Weltkrieg 1914-1918, Düsseldorf, Droste Verlag, 1970, p. 88.

4 Voir Jörn Leonhard, Die Büchse der Pandora. Geschichte des Ersten Weltkriegs, Munich, C. H. Beck, 2014, p. 513 sq.

5 Voir BArch R/8034/II 1451, Brot und Bäckererzeugnisse - Zusatz von Kartoffeln bzw. Kartoffelmehl zum Brot - Verband Deutscher Brotfabrikanten - vom 8. September 1914 bis 5 März 1915, vol. 1, p. 12, article «Das "K"-Brot», $1^{\text {er }}$ déc. $1914, n^{\circ} 610$.

6 Cf. Anne Rasmussen, “L'alimentation rationnelle du soldat”: les sciences de la nutrition à l'épreuve de la guerre", in: Caroline Poulain (dir.), Manger et boire entre 1914 et 1918, Gand, Snoeck, 2015, p. 56-66. 
22 février 1915 et, à partir du 15 mars 1915, dans toute l'Allemagne ${ }^{(7)}$. L'introduction de la carte de rationnement fut un événement commémoré l'année suivante dans la presse $^{(8)}$. Seule cette carte permettait l'achat de pain. Personnalisée, d'une couleur spécifique selon la semaine, elle était composée de tickets, en échange desquels il était possible d'obtenir une certaine quantité de pain. À Berlin, les premières cartes furent distribuées à la population civile par les propriétaires d'appartement et les professeurs volontaires $^{(9)}$.

Le rationnement sonna le clairon de la guerre sur le front de l'arrière, la Heimatfront. Ce fut l'un des effets les plus palpables du conflit à l'arrière. La peur au ventre, les citoyens tournèrent leurs espoirs vers l'État et, à partir de 1915, vers un Lebensmitteldiktator utopique, un pouvoir central dictant les rations, évitant ainsi toute inégalité et apaisant les tensions croissantes entre producteurs et consommateurs ${ }^{(10)}$. Cela revenait à attendre d'un État fédéral, qui n'avait été unifié à grand-peine qu'en 1871, qu'il développe le fonctionnement d'un État centralisé.

La création du Kriegsernährungsamt (KEA) le 22 mai 1916 fut une tentative de centralisation des différentes institutions liées à la répartition des denrées pendant la guerre. Son action se déploya à partir de la capitale où les premières cartes de rationnement furent conçues, servant de modèle aux autres villes allemandes. Mais la déception face à l'inefficacité de cette institution fut immense et s'exprima dans les journaux à partir de 1917. C'est alors que parut le plus grand nombre d'articles portant sur «le manque de pain», avec des titres tels que «Pourquoi n'y a-t-il pas assez de pain?» ou «Diminution de la ration hebdomadaire?» (janvier), "Adieu à nos petits pains» (février), ou encore «Diminution de notre ration de pain» et «Eh bien, M. Bartocki? " ${ }^{(1)}$ (mars). Ils attestent d'une vision critique du système de rationnement et de son inefficacité.

Dès janvier 1917, un autre phénomène notable fut le vol de cartes de rationnement ${ }^{(12)}$. Plusieurs articles l'évoquèrent également en avril et en décembre ${ }^{(13)}$. Mais, alors que les

7 Cf. BArch R/8034/II 1451, Brot und Bäckererzeugnisse (note 5), vol. 2, «Bekanntmachung», 11 mars $1915, n^{\circ} 595$.

8 Ibid., p. 61, «Jubiläum», 25 janv. 1916; BArch R/8034/II 1451, Brot und Bäckererzeugnisse (note 5), vol. 4, p. 69, «Das Jubiläum der Brotkarte. Einführungstag 22. Februar», 21 fév. 1917.

9 Ibid., p. 150, «Kein Brot ohne Brotkartei», 11 fév. 1915; p. 154, «Berlin im Zeichen der Brotmarken», 12 fév. 1915.

10 Voir le concept de Lebensmitteldiktator chez Wolfgang ECKART, «Schweinemord und Steckrübenwinter - Hungererfahrungen und Lebensmitteldiktatur, 1914-1918», in: Medizin, Gesellschaft und Geschichte. Jahrbuch des Instituts für Geschichte der Medizin der Robert Bosch Stiftung, 13 (2013), p. 9-31.

11 BArch R/8034/II 1451, Brot und Bäckererzeugnisse (note 5), vol. 4, p. 28, «Brotmangel», 18 janv. 1917 ; p. 31, «Weshalb mangelt es an Brot», 18 janv. 1917; p. 30, «Die Brotfrage. Verringerung der Wochenration?», 22 janv. 1917; p. 35, «Schrippe Ade», 27 janv. 1917; p. 96, «Die Herabsetzung der Brotration», 29 mars 1917; p. 97, «Die Kürzung der Brotration», 24 mars 1917; p. 98, «Nun, Herr von Bartocki?», 24 mars 1917. Adolf von Bartocki fut le premier président du KEA.

12 Ibid., p. 43, «Brotkartendiebstahl», 28 janv. 1917. Les vols eurent même lieu en dépit de la surveillance armée lors du transfert des cartes de rationnement.

13 Ibid., p. 118, «Brotkartendiebstahl», 27 avril 1917; p. 196, «Ein ganzer Wagen mit Brotkarten gestohlen», 12 déc. 1917; R/8034/II 1451, Brot und Bäckererzeugnisse (note 5), vol. 5, p. 2, «Klau von Brotkarten und Schwindel», 30 déc. 1917. 
annonces gouvernementales occupaient des pages entières, ce type d'événement pouvait passer inaperçu. En effet, il était évoqué en seulement quelques lignes, au sein de très modestes articles, sans titres détachés du corps du texte. En revanche, les peines encourues étaient très précisément énoncées afin de dissuader les lecteurs de commettre de telles infractions et de simuler un contrôle en réalité quasiment inexistant. En dépit de peines pouvant s'élever jusqu'à cinq mois de prison pour le vol ou la vente illégale de tickets déjà utilisés, un marché noir de tickets de rationnement vit le jour ${ }^{(14)}$.

Par ailleurs, dès l'année 1915, les autorités tentèrent, avec l'aide de médecins tels que le docteur Rubner, de trouver de nouvelles méthodes de fabrication. La description de ces efforts se fit de plus en plus fréquente dans la presse. Un pain constitué en partie de sang d'abattoir, appelé Blockbrot, Kraftbrot ou Globulinbrot, permettant d'économiser 20 à $25 \%$ de farine, apparut parmi les premières propositions le 25 janvier $1915^{(15)}$. Par ailleurs, les archives administratives du Reich (série R86 du ministère de la Santé) témoignent d'un changement d'attitude lors de l'année 1916, dont les répercussions furent considérables en 1917: alors que certains ersatz nuisibles à la santé furent d'abord interdits, dont la farine "d'écorce, de paille et d'épeautre " ${ }^{(16)}$ illégalement utilisée en mai $1916^{(17)}$, celle-ci fut finalement autorisée le 28 septembre de la même année. Bien que les conséquences sur la santé publique fussent connues, cette décision révélait non seulement un relâchement de la contrainte législative, mais aussi la grande importance accordée à la préservation du pain pour les populations. La préoccupation première n'était pas la santé publique mais le « remplissage inoffensif des estomacs » ${ }^{(18)}$, tel que les politiques le formulèrent dans leurs correspondances, car l'effort de guerre ne devait pas faiblir. Préserver le moral des civils passait avant tout. L'assouplissement de la législation prenait racine dans la relation implicite qui était établie par les autorités entre le pain, symbole de la nourriture dans son ensemble, et les mouvements révolutionnaires. Elle fut précédée de moult hésitations de la part des ministres. Ainsi, le ministre de la Santé ne souhaitait pas que toutes ces informations soient divulguées, de peur qu'elles ne soient «exploitées par nos ennemis afin de confirmer l'idée d'une Allemagne à l'agonie sous l'emprise de la famine» et qu'elles nuisent aussi «au maintien de la confiance et du courage du peuple allemand ${ }^{(19)}$.

Le pain de la marque "Eckhoff», au sujet duquel le ministère de l'Alimentation archiva les publicités et les articles de mars $1916^{(20)}$, est le produit de l'introduction de

14 Ibid., p. 160, «Kuddelmuddel», 2 fév. 1915.

15 Voir BArch R/8034/II 1451, Brot und Bäckererzeugnisse (note 5), vol. 1, p. 98, «Blutbrot», 25 janv. 1915; R86/ 3286, vol. 3 : Massen- und Unterernährung im Krieg (1914-1918), vom 15. Dezember bis 31. März 1915, article «Blut als Nahrungsmittel», Sonderabdruck der Bonner Zeitung, 11 mars 1915; «Blockbrot», 18 mars 1915.

16 BArch R86/034/ II 1451, Brot und Bäckererzeugnisse (note 5), lettre de l'entreprise Minerva-Werke E.ઐM. Gränitz (fabriquant de farine) au ministre de l'Intérieur, le 9 octobre 1915.

17 Voir BArch R86/ 5441, Kriegsbrot, Kartoffelbrot (K-Brot), article «Brotfälschungen», 5 mai 1916; article «Brotfälschungen», 11 mai 1916.

18 BArch R86/ 3286, Massen- und Unterernährung im Krieg (note 15), lettre du directeur du ministère des Affaires étrangères H. Johannes au ministre, 14 janv. 1915.

19 Ibid. Ces passages étaient barrés dans le brouillon de sa lettre mais témoignent de ses inquiétudes.

20 Voir BArch R86/ 5441, Kriegsbrot, Kartoffelbrot (note 17), «Warnung vor Eckhoff-Brot», 22 mars 1916. 
ces nouvelles farines incongrues, à base d'écorce et de bois. Une affiche publicitaire lui attribue des vertus quasi miraculeuses face à tous les maux de l'époque: sous-nutrition, faiblesse, fatigue, tuberculose, etc. Or, les autorités n'avaient alors pas encore autorisé la farine de paille. L'affiche révèle donc la difficulté à mettre en application la législation existante. Enfin, en avril 1917, le lieutenant Bauer confirme: «Nous avons fait de graves erreurs en matière de fixation des prix, de distribution des denrées et lors des tentatives pour augmenter la production ${ }^{(21)}$.

\section{B. L'absence du pain et la remise en question du Burgfrieden}

Afin de cerner les ruptures qui sont apparues en 1917, il est aussi nécessaire de prendre en compte la représentation d'un pays comme forteresse assiégée ${ }^{(22)}$. À l'intérieur devait être préservé le Burgfrieden, l'équivalent de l'union sacrée ${ }^{(23)}$ et, littéralement, «la paix à l'intérieur des remparts». En mars 1917, d'après le ministre bavarois de la Guerre, l'Allemagne devait faire face à l'«épreuve d'un siège quasi inégalée» ${ }^{(24)}$. Dès 1914, la théorie de l'encerclement, présentée comme préméditée par les Anglais pour affamer le peuple allemand, fut notamment diffusée grâce à l'affiche intitulée "L'Allemagne face à un monde d'ennemis » ${ }^{(25)}$. Or, un seul aliment était mentionné sur cette affiche et c'était le pain:

«Soyez respectueux vis-à-vis du pain car, dans ce cas, vous en aurez toujours assez, peu importe la durée de la guerre. Éduquez vos enfants dans ce sens, ne négligez pas un morceau de pain s'il n'est plus frais. Ne coupez pas plus de tranches de pain que ce que vous êtes en mesure de consommer. Pensez toujours à nos soldats sur le champ de bataille et dans des lieux reculés qui, souvent, seraient heureux s'ils avaient le pain que vous gaspillez. Mangez du pain de guerre. Il est reconnaissable par la lettre ' $K$ '. Il rassasie autant qu'un autre pain. Si tout le monde le mange, nous ne devrons pas nous soucier de la question du pain. [...]» ${ }^{(26)}$.

Ces lignes étaient typiques d'un discours patriotique officiel, relayé entre 1914 et 1915 par de nombreux journaux et dans les introductions des livres de recette: le pain se mua alors en arme de la Heimatfront. La comparaison avec les soldats octroyait à la ménagère un rôle actif, se rapprochant de celui des munitionnettes. Même les enfants étaient appelés à jouer un rôle, le gaspillage du pain par les écoliers devenait un sujet récurrent dans la presse ${ }^{(27)}$. Néanmoins, ces discours moralisateurs se raréfièrent au cours du conflit. Au début, les autorités comptaient sur l'autocensure des

21 Matthias/Welcker (dir.), Quellen zur Geschichte des Parlamentarismus (note 3), vol. 1, p. 718.

22 Voir à ce propos la thèse en cours de Lise GALAND, "L'Allemagne impériale et le spectre de l'encerclement entre 1906 et 1914: diffusion, circulation et transformation d'une notion dans l'espace public ", à l’université Paris-Sorbonne sous la direction d'Hélène Miard-Delacroix et d'Olivier Forcade.

23 Ce terme a une signification politique et sociale. Ici, nous utiliserons sa signification étendue à la société telle que le fit Guillaume II lors de son discours du 4 août 1914, dans le sens d'un consensus pour rester uni et maintenir l'effort de guerre.

24 Matthias/Welcker (dir.), Quellen zur Geschichte des Parlamentarismus (note 3), vol. 2, p. 700.

25 BArch R86/ 3286, Massen- und Unterernährung im Krieg (note 15), Affiche «Deutschland steht gegen eine Welt von Feinden ", $1^{\text {er }}$ juin 1914.

26 Ibid.

27 Voir BArch R/8034/II 1451, Brot und Bäckererzeugnisse (note 5), vol. 4, p. 70, "Schule und Kirche als Helfer unserer Brotgetreideversorgung», 3 fév. 1916. 
journalistes ${ }^{(28)}$, mais le système n'était pas toujours efficace, du fait des structures fédérales, des particularismes et des directives floues. L'enquête lancée en 1917 par l'Office central de censure du département IIIb sur les censeurs eux-mêmes montra le peu de confiance que les autorités militaires accordaient à l'autocensure: ces autorités se méfiaient du peuple allemand, et même de leurs propres fonctionnaires qui souffraient alors particulièrement des pénuries.

Le 11 novembre 1916, les citoyens tentèrent d'atteindre le Reichstag et le château de l'empereur en scandant «nous voulons la paix et du pain!», à l'image des révolutionnaires français de $1789^{(29)}$, le pain faisant office de symbole de la nourriture en général. Dès le 10 janvier 1917, le chancelier du Reich, Georg Michaelis, publia un article adressé au peuple, évoquant les grandes disparités que comportait, «bien entendu », le rationnement des céréales. Il tenta de réconcilier et d'apaiser les citadins souffrant du rationnement et les ruraux victimes des confiscations ${ }^{(30)}$. En même temps, il propagea l'idée (fausse) d'après laquelle les inégalités ne reposaient pas sur un manque quantitatif de denrées, mais sur les précautions préventives de rationnement ${ }^{(31)}$, afin d'éviter une panique mettant en péril le consensus de guerre et ainsi la stabilité de l'État. Le 17 janvier 1917, la pression exercée par l'arrière à cause des pénuries s'intensifia ${ }^{(32)}$. En mars, les notes prises lors de la réunion des présidents des bureaux de censure régionaux à propos des articles sur la révolution russe et l'alimentation prirent un ton alarmiste et pessimiste: comment remettre la population dans le droit chemin si, jusque-là, la presse n'avait pas encore été censurée sur les questions d'alimentation et si détresse et privations avaient atteint la classe moyenne ${ }^{(33)}$ ? La solution alors trouvée fut la distribution de tracts de propagande accompagnés d'une carte de pain ${ }^{(34)}$.

Mais ce fut en vain car l'agriculture et l'arrière avaient été négligés par rapport au front et le pain avait été tant valorisé qu'une volte-face soudaine n'était plus possible. C’est pour cela qu'il est nécessaire de revenir sur les représentations du pain en cours dans les années précédentes. Dès 1915, le Kaiser lui-même dit consommer du K-Brot afin de montrer l'exemple ${ }^{(35)}$. Sur une affiche de 1915 intitulée Wir lassen uns nicht aushungern (nous ne nous laisserons pas mourir de faim), les enfants furent dépeints

28 Ils étaient appelés à passer sous silence toute information stratégiquement et militairement nuisible à l'Allemagne.

29 Voir Matthias/Welcker (dir.), Quellen zur Geschichte des Parlamentarismus (note 3), vol. 1, p. 294.

30 Voir BArch R/8034/II 1451, Brot und Bäckererzeugnisse (note 5), vol. 4, p. 25, "Reichsgetreide jetzt und später», 10 janv. 1917. En effet, même la farine humide auparavant destinée aux bestiaux devait être remise aux autorités.

31 Voir BArch R/8034/II 1451, Brot und Bäckererzeugnisse (note 5), vol. 1, p. 2, «Kriegsbrot», 8 déc. 1914.

32 Ibid., p. 659.

33 Ibid., p. 159.

34 Ibid., p. 808

35 BArch R86/ 3286, vol. 3 : Massen- und Unterernährung im Krieg (note 15), « Kriegsdienst im Haushalt, ein Wort an die deutschen Frauen", Hauptvorstand des vaterländischen Frauen-Vereins, (non daté). Néanmoins, même si cela fut le cas, le pain de guerre (K-Brot) que celui-ci consommait devait être de bien meilleure qualité que celui consommé par la majorité de la population, le problème n'étant pas en premier lieu l'ajout de pommes de terre, mais celui de farines non comestibles. BArch R/8034/II 1451, Brot und Bäckererzeugnisse (note 5), vol. 1, p. 34 bis, «Der Ruf nach dem K-Brot» et «Der Kaiser ißt Kriegsbrot», 5 janv. 1915. 
en guerriers de la Heimatfront ${ }^{(36)}$, tenant une tranche de pain, l'arme de leur lutte. En 1916, le pain de guerre fut défini comme l'«arme la plus forte qui soit» ${ }^{(37)}$ à travers des publications, mais aussi à travers l'iconographie. Celle-ci fut véhiculée par des objets rappelant les activités quotidiennes et rythmant les journées, comme l'assiette patriotique portant l'inscription «Besser K-Brot als Kaa Brot» du repas, la médaille pour le mérite intitulée «Ohne Brot kein Sieg», qui était attribuée pour des dons liés à l'alimentation des soldats ${ }^{(38)}$, ou la bande-dessinée retraçant le conte de Hansel et Gretel retrouvant leur chemin grâce à des miettes de pain ${ }^{(39)}$. Ainsi, le pain était représenté comme un élément salvateur de la Heimat, l'espace protecteur où les Allemands trouvaient leur enracinement culturel.

Bien qu'ayant été une pratique courante, aussi bien en France qu'en Grande-Bretagne ou aux États-Unis ${ }^{(40)}$, la tendance à faire du pain une arme matérielle et symbolique de l'arrière eut des conséquences particulièrement graves en Allemagne. Les symboliques religieuses étant déjà profondément ancrées au sein de la société, les paroles patriotiques ne firent que renforcer le lien entre le pain et la nourriture, le pain et le corps ou le pain et la vie. Lorsque, au cours de l'hiver 1916/1917, le pain disparut quasiment des repas ou se dégrada à tel point que sa consommation devint souvent nocive, l'univers représentatif et symbolique dont il était l'appui en pâtit également. Si la survie était, dans l'imaginaire populaire, inexorablement liée au pain en tant que base culturelle et nutritionnelle de l'alimentation, comment en son absence préserver le moral de guerre? Comment l'alimentation, le repas du soir appelé Abendbrot, littéralement pain du soir, et la vie elle-même pouvaient-ils être envisagés sans pain ? La disparition d'un pain qualitativement et gustativement acceptable pour le plus grand nombre contribua ainsi à la remise en question du Burgfrieden.

\section{Une sensibilité aiguisée: le pain, un luxe?}

Nous venons d'évoquer le contexte politique et social des grandes ruptures de l'année 1917 concernant le pain ainsi que les premiers signes de ces ruptures. Des ruptures plus discrètes, mais peut-être plus profondes, et s'incarnant dans de nouvelles recettes, pratiques, gestes et perceptions en lien avec le pain quotidien les accompagnèrent: nous allons les évoquer maintenant.

36 «Wir lassen uns nicht aushungern» (nous ne nous laisserons pas affamer), affiche de propagande concernant le manque de nourriture en Allemagne, lithographe Friedrich Kaskeline, éditeur Verein für Kindervolksküchen und Kinderhorte (association des soupes populaires pour enfants et garderies), 1915, 30 x 24,5 cm, Deutsches Historisches Museum (DHM), Berlin, 1989/1344.

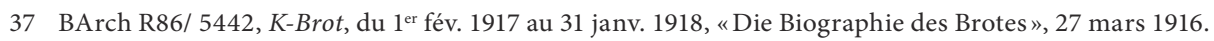

38 Médaille «Ohne Brot kein Sieg» (sans pain, pas de victoire), 1,9 cm, DHM, Berlin.

39 Voir «Hänsel und Gretel und das K-Brot», 1915, dans: Anne Roeнr конL, «Schlachtfeld Heimat», in: Christine BeIl (et al.), Der Erste Weltkrieg, Berlin, Rowohlt, 2004, p. 165 sq, p. 184.

40 Voir les différents slogans: en Angleterre: «Bread is the key to victory»; aux États-Unis: "Save a loaf a week - help win the war»; en France: "Économisez le pain. Coupez-le en tranches minces... et utilisez toutes les croûtes pour le souper» ou bien «Ne pas gaspiller le pain est notre devoir». 


\section{A. Rareté du pain, dissensions et Dolchstoßlegende}

Même dans le milieu de la bourgeoisie intellectuelle, par exemple au sein de la famille Hampe, dès septembre 1916, la ration de pain par personne n'était plus que de 280 grammes par jour ${ }^{(41)}$ et la famille ressentait la faim continuellement. Pour Charlotte, l'épouse de Karl Hampe, les pénuries furent particulièrement difficiles à supporter lorsque, à Heidelberg, les tickets de rationnement furent épuisés et que «les réclamations en pain des enfants ne purent être satisfaites " ${ }^{(42)}$. Les navets constituaient même, d'après une note du 25 février 1917, leur nourriture principale puisque «les pommes de terre devaient être économisées. Les Semmeln [petits pains] ne sont plus fabriqués » ${ }^{(43)}$. Peut-être était-ce une réaction à la lecture de la presse qui encourageait la population à inventer de nouveaux ersatz pour le pain et prônait «l'utilisation du gland à des fins de consommation » ${ }^{(4)}$ (6 février 1917) et "l'utilisation de betteraves pour l'allongement de la pâte à pain » ${ }^{(45)}$ (30 mars). Ce dernier article contenait une directive du KEA indiquant que le navet devait remplacer la pomme de terre lors de la fabrication du pain, ainsi qu'une recette à cet effet.

Karl Hampe, professeur d'histoire à l'université de Heidelberg, chercha alors à dédramatiser la situation. D’après lui, «le plaisir [culinaire] est très relatif», car l'habitude de mets raffinés diminue la capacité de leur appréciation ${ }^{(46)}$. Sa réflexion sur le goût devait mener à une relativisation de la situation et à la valorisation d'un contrôle sur soi et son corps. Cette orientation apparaissait déjà dans la presse de 1916 qui mettait en valeur les avantages de la pénurie pour la santé publique car elle entraînait une perte de poids et une diminution des excès. On retrouve donc sous la plume de l'historien le discours de la presse qui encourageait l'Alltagsheroismus ${ }^{(47)}$. Hampe souhaitait peut-être ainsi adoucir la réalité: le contexte dans lequel sa famille se trouve au début de l'année 1917 est clairement en rupture par rapport aux années précédentes.

Depuis l'été 1916 en effet, les idées de Gemeinschaft, de communauté et de Burgfrieden se désagrégeaient, notamment au sein des milieux ouvriers et de la bourgeoisie lettrée ${ }^{(48)}$. Les critiques contre les «profiteurs» de guerre venaient remettre en cause l'esprit en apparence harmonieux de 1914. On cherchait des responsables pour le rationnement inégal et les infrastructures étatiques défaillantes. En 1917, la grande précarisation du quotidien fut le coup de grâce pour bon nombre d'écrits patriotiques. Timidement, et avec peu de moyens, les autorités tentèrent tant bien que mal de les faire revivre, mais sans grand résultat. Le 4 avril, la diminution des rations de pain servit même d'argument au chancelier du Reich, Theobald von Bethmann-Hollweg,

41 Voir Karl Haмpe, Kriegstagebuch 1914-1919, Munich, Oldenbourg, 2004, p. 459.

42 Ibid., p. 429.

43 Ibid., p. 129.

44 BArch R86/ 5442, K-Brot (note 37), « Nutzbarmachung der Eichel für die Volksernährung », 6 fév. 1917. BArch R86/ 5442, K-Brot, ibid., «Verwertung von Rüben als Brotstreckungsmittel», 30 mars 1917. K. HAMPE, Kriegstagebuch 1914-1919 (note 41), p. 507. Expression signifiant «héroïsme au quotidien» utilisée par Folker Reichert: voir Folker REICHERT, Eike Wolgast (dir.), Karl Hampe - Kriegstagebuch 1914-1919, Munich, Oldenbourg, 2007.

48 Gerhard Hirschfeld, Gerd Krumeich, Irina Renz (dir.), Enzyklopädie Erster Weltkrieg, Paderborn, Ferdinand Schöningh, 2009, p. 19. 
pour obtenir l'annonce du droit de vote des femmes dans le message de Pâques de l'empereur, contre l'avis du commandement suprême des armées ${ }^{(49)}$.

Alors que de nombreuses révoltes de la faim avaient éclaté en 1915 et en 1916, révoltes représentées notamment dans les œuvres de Franz Wimmer et de Käthe Kollwitz $^{(50)}$, l'épuisement paralysa ces révoltes à la fin de l'hiver 1916/1917. Les énergies contestataires ne réapparurent qu'au printemps de l'année 1917 et en janvier $1918^{(51)}$, sous l'influence de la révolution russe ${ }^{(52)}$. Elles furent encouragées par le sentiment que la rupture des habitudes alimentaires avait été trop brutale, rupture qui mettait en péril la vie elle-même. La faim entraîna l'augmentation de la mortalité néo-natale et la recrudescence de la tuberculose et des anémies. La taille et le poids moyens des enfants des villes diminuèrent ${ }^{(53)}$. La dégradation du moral à l'arrière et les révoltes furent ensuite instrumentalisées par les autorités militaires afin de justifier la légende $\mathrm{du}$ «coup de poignard dans le dos» («Dolchstoßlegende») ${ }^{(54)}$.

\section{B. De nouvelles pratiques et la valeur du pain}

Le 6 juin 1917 parut un article sur les «Ersatz pour le pain et la farine non comestibles» ${ }^{(55)}$ et le 3 juillet un article sur «le pain de guerre nuisible à la santé - une conséquence de la pénurie de blé?» ${ }^{(56)}$. L'auteur mettait en cause la mauvaise qualité du pain, à l'origine selon lui de maladies, surtout au sein des populations pauvres, pour lesquelles le pain constituait l'aliment principal. D'après cet article, de nombreuses plaintes étaient adressées aux boulangers pour cause de livraison de pains non comestibles. «La pénurie de blé est-elle vraiment si importante qu'une farine de si mauvaise qualité puisse être livrée?» : la question suggère que le journaliste ne peut se résoudre à croire à une véritable disette.

49 Voir Matthias/Welcker (dir.), Quellen zur Geschichte des Parlamentarismus (note 3), vol. 1, p. 703.

50 Voir Franz Wimmer, «Wir wollen Frieden», dessin au fusain, 1916, in: Ch. Beil, Der Erste Weltkrieg (note 39), p. 189; Silke FEHLEMANN, «Anstehen nach Lebensmitteln» (http://centenaire.org/fr/ print/5183, le 31.08.2014); Belinda Davis, Home Fires Burning: Food, Politics, and Everyday Life in World War I Berlin, Chapel Hill, University of North Carolina Press, 2000 (voir le chap. «Battles over Butter», p. 76); Doris KaChulle (dir.), Die Poehlands im Krieg. Briefe einer sozialdemokratischen Bremer Arbeiterfamilie aus dem 1. Weltkrieg, Cologne, Pahl-Rugenstein (coll. «Kleine Bibliothek», 246), 1982, p. 129 (Anna Pöhland décrit dans son journal une révolte à laquelle elle a contribué en juin 1916, au cours de laquelle le pain est brandi, matériellement et symboliquement); MAtThias/WeLCKeR (dir.), Quellen zur Geschichte des Parlamentarismus (note 3), vol. 1, p. 194 (le 30 juin 1917 eut lieu un soulèvement d'un grand nombre d'adolescents ayant rejoint la ligue spartakiste [Spartakusbund] et, la même année, 200 personnes furent tuées ou blessées pendant une manifestation).

51 Voir Gustavo Corni, «Hunger», in: Hirschfeld/Krumeich/Renz (dir.), Enzyklopädie Erster Weltkrieg (note 48), p. 565.

52 Voir le poème d'Erwin Hoernle, communiste, daté de 1918: «Nous réclamons la paix, nous réclamons du pain, / le monde est mort, / Nous sommes enchaînés, sommes ligotés, / La faim et la maladresse nous font souffrir, / La misère nous tue.» Je remercie Nicolas Patin de m’avoir indiqué cette source. BArch R86/ 3281, «Der deutsche Hunger», Frankfurter Zeitung, n² 262, 10 avril 1920.

54 Ibid., p. 159.

55 BArch R86/ 5442, K-Brot (note 37), «Ersatz für ungenießbares Brot und Mehl», 6 juin 1917.

56 BArch R86/ 5442, K-Brot, ibid., «Das gesundheitsschädliche Kriegsbrot - eine Folge der Weizenknappheit?», 3 juillet 1917. 
Bien que le pain de guerre allemand ait pu paraître plus nourrissant que le pain blanc français, les ersatz non comestibles qui y étaient introduits semblaient entraîner des maladies intestinales empêchant l'assimilation des aliments. Anne Steinmetz, née en 1896 et domestique en Sarre, supposa dès 1915 que l'ajout de farine de paille était à l'origine des nausées provoquées par le pain chez les personnes de son entourage ${ }^{(57)}$. Le pain de guerre n'empêcha pas non plus les œdèmes causés par la sous-nutrition à partir d'avril $1917^{(58)}$. Le journal des sœurs Clara et Joséphine Bohn, originaires d'Ingersheim en Alsace, rédigé à onze et treize ans dans un milieu modeste, fait aussi référence à un pain consommé "plus mauvais qu'aucun autre pendant la guerre». Elles le relient à l'invention d'une farine nommée Kieselguhr ou Kieselmehl (farine de gravier ${ }^{(59)}$. La description de cette farine reprend presque mot pour mot certains éléments d'un article paru dans la presse le 10 octobre $1917^{(60)}$, tels que la couleur, la provenance et l'utilisation de cette farine pendant la guerre de Trente ans. Le journal des sœurs Bohn, relu par leur père, peut être perçu comme un exercice d'écriture, mais dévoile aussi l'influence de la presse et l'ignorance, non seulement des enfants, mais aussi des parents, concernant la véritable composition du pain.

En ville, les écrits d'enfants issus du milieu ouvrier rapportent qu'ils durent très jeunes remplacer leur mère dans les files d'attente, notamment pour obtenir du pain. C'est dans cet espace, ainsi qu'à table, que la relation à cet aliment s'incarna pour eux le plus distinctement, par la vue, le goût, mais aussi l'odeur et le toucher. Ainsi, Otto Rees, né en 1910 à Fribourg-en-Brisgau et issu d'un milieu ouvrier ${ }^{(61)}$, en fit l'expérience. Son père ayant été mobilisé dès 1914, sa mère devait subvenir aux besoins de la famille: elle travaillait dans une fabrique de grenades, cousait des sacs de sable, distribuait le courrier et plantait des pommes de terre dans un petit jardin ${ }^{(62)}$. Rees se souvient, grâce au modelage de la mie, du toucher du pain contenant, d'après lui, de la sciure de bois ${ }^{(63)}$. Bien qu'ayant grandi dans une famille aisée, son père étant juge, Ernst Glaeser remarqua également la consistance visqueuse et pâteuse du pain décrite par la presse en août $1917^{(64)}$. Cette consistance permet au personnage principal de son roman autobiographique de modeler le pain en forme de figurines au lieu de le consommer ${ }^{(65)}$.

Les citadins eurent également recours aux Hamsterfahrten, les voyages ponctuels à la campagne permettant d'acheter ou de troquer des denrées ${ }^{(66)}$. Tout soutien des zones

57 Voir TArch, Journal intime d'Anna Steinmetz (1896-1951), p. 15.

58 Voir Leo Lippmann, Mein Leben und meine amtliche Tätigkeit, Hambourg, Christians, 1964, p. 228.

59 TArch, Journal intime de Joséphine et Clara Bohn, p. 41.

60 Voir BArch R/8034/II 1451, Brot und Bäckererzeugnisse (note 5), «Eßbare Erde», Zeitung für Nahrungsmittel, $\mathrm{n}^{\circ} 237,10$ oct. 1917.

61 Voir TArch 921, Journal intime d'Otto Rees, Kein Engel - kein Held, chap. "Vergnügliche Laubernte», Préface.

62 Ibid., p. 5.

63 Ibid., p. 8.

64 Cette description se trouve dans BArch R/8034/II 1451, Brot und Bäckererzeugnisse (note 5), vol. 4, p. 155, «Fadenziehendes Brot», 5 août 1917.

65 Voir Ernst GLÄSER, Jahrgang 1902: Roman, s.1., Bertelsmann Lesering, 1961, p. 261.

66 Voir K. Hамpe, Kriegstagebuch 1914-1919 (note 41), p. 555 et 558. 
rurales était le bienvenu. Otto Schneider, né en 1905 dans une famille modeste de Plauen, une petite ville de l'Est de l'Allemagne, rendait visite à ses tantes à la campagne et se rappelait de la tranche de pain beurré qui lui avait procuré un plaisir particulier sur le chemin du retour ${ }^{(67)}$. Ayant grandi à Fribourg dans une famille souffrant des pénuries, envoyé à la campagne en Suisse pour reprendre du poids et pesé tous les jours, Otto Rees se souvient lui aussi de cette expérience particulière du pain beurré, bien différent de leur "pain noir contenant de la farine de bois » ${ }^{(68)}$. Il se rappelait que "le pain pesait plus lourd que de l'or» ${ }^{(69)}$. La valeur ainsi accordée au pain s'incarnait aussi dans le geste «de la plupart des femmes» de mettre le pain sous clé, « dans la boîte à pain habituelle» ${ }^{(70)}$, afin d'empêcher les enfants de se servir.

À la campagne, à partir de 1915, le pain était fabriqué au sein du foyer familial et cuit dans le four du boulanger, car on commençait à se rendre compte que le pain acheté provoquait des nausées ${ }^{(71)}$. Il était souvent consommé émietté dans un café, un thé ou une soupe, parfois, en temps de grande pénurie, sans garniture, sous forme de trockenes Brot (pain sec) ${ }^{(72)}$. Les tickets de pain servaient même de supports afin d'y coller d'autres tickets de rationnement ${ }^{(73)}$. Mais même sous forme de ticket, le pain matérialisait quelque chose d'incomparable, ce dont Otto Rees se souvient avec émotion: après l'école, la priorité absolue des enfants, après avoir obtenu de l'argent de leurs parents, était de "pouvoir présenter sans attendre [au boulanger] la partie du coupon destinée au pain", coupon que leurs "petites mains crispées conservaient telle une pierre précieuse ${ }^{(74)}$. La préservation de quelques miettes dans un cas, d'une tranche ou d'un morceau dans d'autres, fait aussi écho à un attachement irrationnel au pain ${ }^{(75)}$. Une pratique courante pour compenser le manque d'aliments de base, et notamment de pain, était la collecte de marrons, glands ${ }^{(76)}$ et faînes dans les forêts par les écoliers. Ceux-ci pouvaient ensuite recevoir «de la main de l'instituteur un ticket de rationnement pour une quantité en tout et pour tout de cent grammes de pain", ticket que l'instituteur détachait "avec la plus grande méticulosité» ${ }^{(77)}$. En bord de mer, d'après le journal d'Ina Cartensens, une féministe engagée, nommée au sein de la commission d'alimentation de la petite ville de Husum, au nord de l'Allemagne, le pain était remplacé par des recettes d'urgence, entre autres par des fricadelles. Ces dernières étaient composées de fruits de mer passés au hachoir et mélangés à de la farine

67 Voir TArch 2162, Journal intime d'Otto Schneider (1905-1978), chap. «Schwere Last auf schwachen Schultern", p. 4.

68 TArch 921, Otto Rees, Kein Engel - kein Held (note 61), p. 6.

69 Ibid., p. 3.

70 TArch 2162, Journal intime d'Otto Schneider (note 67), p. 4.

71 Voir TArch 1020, Journal intime d'Anna Steinmetz (1896-1951), p. 15.

72 TArch 2016-1, Souvenirs d'enfance de Frieda Anna Hiller (1909-1986), 1962, p. 4.

73 Voir Leo Lippmann, Mein Leben und meine amtliche Tätigkeit (note 58), p. 231.

74 TArch 921, Journal intime d'Otto Rees, Kein Engel - kein Held (note 61), chap. «Vergnügliche Laubernte», p. 3. Voir image issue du Imperial War Museum: EPH 5677, Pain «KK». Voir BArch R86/ 5442 (note 37), «Nutzbarmachung der Eichel für die Volksernährung», 6 fév. 1917. TArch 2162, Journal intime d'Otto Schneider (note 67), chap. «Schwere Last auf schwachen Schultern", p. 2. 
ou à des flocons d'avoine. Bien que la levure ajoutée en tant qu'ersatz de farine donnât au pain un goût si désagréable qu'il "provoquait toujours le même dégoût», en dépit de l'habitude prise "de manger tout ce qui était comestible», il faisait constamment partie des aliments consommés ${ }^{(78)}$. D’après Otto Schneider, «ce qui était de loin le plus important [...] était la sensation de satiété pour laquelle la dégradation du goût était le prix que l'on était prêt à payer» ${ }^{(79)}$. La faim et «les papilles rendues insensibles » ${ }^{(80)}$ induisaient l'acceptation de denrées exécrables. Cela confirme l'efficacité, au moins temporaire, d'une politique prête à accepter des ersatz non comestibles, estimant apparemment que le soutien du moral de la population allemande était plus important que la préservation de sa santé.

\section{Déceptions et nouveaux espoirs: une difficile fin d'année}

La politique des premières années de guerre mettait l'accent sur la préservation et l'idéalisation du pain en tant qu'arme de la Heimatfront. La sensibilité accrue qui en résultait mena à l'érosion du Burgfrieden et du soutien de l'opinion allemande à la guerre. Les normes et a priori sociaux qui se formèrent alors autour du pain furent cependant bouleversés en fin d'année et occasionnèrent de nouvelles déceptions à l'échelle des individus, mais aussi un défaitisme général.

\section{A. La politique du laisser-faire: un défaitisme général?}

En août 1917, la censure s'effrita. Plusieurs articles parus entre le 5 août et le 8 septembre portèrent sur la mauvaise qualité du pain et les maladies qui, d'après les auteurs, en résultaient ${ }^{(81)}$. Un article du 12 septembre tenta néanmoins de rassurer la population ${ }^{(82)}$. D'après ses auteurs, le pain n'était en rien la cause de maladies. En fin d'année s'opéra, en même temps qu'une très nette dégradation des conditions matérielles de vie, une transformation de ce qui n'était jusque-là qu'un débat en un problème majeur de l'arrière. Des livrets à l'attention des agriculteurs et des citadins eurent pour but de réconcilier les deux parties qui étaient toutes les deux persuadées de la malhonnêteté de l'autre. Les citadins devaient être convaincus des efforts fournis par les populations rurales productrices, ainsi que de leur volonté de partager et non de conserver ce qu'elles produisaient. De leur côté, les populations rurales devaient comprendre en quoi la confiscation de leur production était nécessaire à la survie de la population urbaine.

Une affiche confirme l'effort des autorités pour favoriser l'empathie, dans le but de rétablir l'idée de Gemeinschaft et l'entraide entre ruraux et citadins: une famille est rassemblée autour d'une table, l'ensemble des regards est tourné vers la boîte de

78 TArch 87 / II, Ina und Hans Cartensens. Familienchronik, p. 93; R/8034/II 1451, Brot und Bäckererzeugnisse (note 5), vol. 2, p. 184, «N-Brot», 26 sept. 1915. Les navets séchés servaient d'ersatz de café et les haricots d'ersatz de farine pour les gâteaux.

TArch 2162, Journal intime d'Otto Schneider (note 67), chap. «Heimatfront 1914-18», p. 4.

81 Cf. BArch R/8034/II 1451, Brot und Bäckererzeugnisse (note 5), vol. 4, p. 155, «Fadenziehendes Brot», 5 août 1917; p. 163, «Kriegsbrot und Zahnfäule», 13 août 1917; p. 154b et 156, « Kriegsbrot und Darmkrankheiten », 4 août 1917; p. 168, «Kriegsbrot und Darmkrankheiten» (sept. 1917 ?).

82 Cf. BArch R/8034/II 1451, Brot und Bäckererzeugnisse (note 5), vol. 4, p. 169, «Die Beschaffenheit unseres Brotes», 12 sept. 1917. 
pain vide qui se trouve dans les frêles mains d'une mère dont les traits expriment la fatigue et l'épuisement ${ }^{(83)}$. Par son absence, ainsi que par les regards portés sur le vide douloureux, le pain prend ici une signification particulière. Il est placé au centre, au croisement des regards et des corps qu'il relie. Les hommes politiques tentaient ainsi, à travers des appels à l'esprit patriotique, de pallier la remise en cause du ciment social, tout en intégrant implicitement le discours religieux, celui du partage du pain pratiqué par Jésus et ses disciples. Le message politique véhiculé est d'autant plus fort qu'il croise concret et abstrait, explicite et implicite. Mais dans l'ensemble, les écrits encourageant le moral de l'arrière se faisaient beaucoup plus rares. Le silence même des autorités trahissait une baisse de moral, même dans les hautes sphères de la politique et de l'administration. L'année 1917 fut rude et le défaitisme planait.

\section{B. Souffrances du quotidien: des mours et des corps atteints}

En dépit d'un été ayant atténué la rupture due à l'absence de pain grâce à la production maraîchère ${ }^{(84)}$, les traces des pénuries étaient encore frappantes à l'automne, d'autant plus que l'affaiblissement des corps n'était parfois apparu pour la première fois qu'au cours même de l'été. Margarethe Schützinger, née en 1863 et issue d'une famille de boulangers, avait fui pour Bayreuth les pénuries de Berlin qu'elle évoquait dans son journal à partir de février 1917. Elle fait état de douleurs aiguës des articulations à partir du 26 juin 1917. Le 15 juillet, elle notait: "pénuries alimentaires, surtout à Berlin». Puis, le 11 septembre, on lui diagnostiqua des carences alimentaires graves lui permettant de percevoir des rations supplémentaires ${ }^{(85)}$. Ce type de récit se multiplia à partir de la fin de l'été. Elfriede Kuhr, dont la mère dirigeait une école de musique à Berlin et le père était architecte à Dantzig, fut élevée par ses grands-parents à Schneidemühl, l'actuel Pila en Pologne. En 1917, elle avait 14 ans. Le 20 août, elle décrit dans son journal intime le désespoir de sa grand-mère, qu'elle surprend un soir accablée par le manque de nourriture, en pleurs et recroquevillée, la tête sur les genoux, les mains devant le visage, assise sur une caisse de charbon. Un des seuls aliments encore disponibles était un morceau de pain ${ }^{(86)}$. S'exprime alors l'impact marquant de la faim, autant par la précision de la description qu'à travers l'événement lui-même, expérience traumatique dépassant l'aspect quantitatif et qualitatif du pain, tout en plaçant celui-ci au centre de la perception de l'acteur en tant qu'aliment de base et de dernier secours.

Depuis 1915, avec la diminution et la dégradation du pain, les Allemands devaient faire face à un changement radical de leurs habitudes alimentaires. Les articles sur la «révolution du petit-déjeuner» ou intitulés "plus de petits pains et les conséquences» en témoignent ${ }^{(87)}$. L'année 1917 est aussi à voir dans la continuité d'une large campagne

83 Affiche de Heinrich HöNCH, «Bauern tut eure Pflicht! Die Städte Hungern» (Paysans faites votre devoir, les villes ont faim!), Munich, R. Schumann, Graph. Werkstätte, 1919.

84 Voir K. Haмpe, Kriegstagebuch 1914-1919 (note 41), p. 559; et TArch. 2162, Journal intime d'Otto Schneider (note 67), chap. «Heimatfront 1914-18», p. 3.

85 Voir TArch 877 / I.1, Journal intime de Margarethe Schützinger, notes du 11 sept. 1917.

86 Voir Jo Minaly, ...da gibt's ein Wiedersehn! Kriegstagebuch eines Mädchens 1914-1918, Fribourg-enBrisgau, Kerle, 1982, p. 290.

87 BArch R/8034/II 1451, Brot und Bäckererzeugnisse (note 5), vol. 1, p. 84, "Frühstückrevolution», «Brötchens Ende», 17 janv. 1915. 
sur les avantages du pain de seigle sur le pain de blé ${ }^{(88)}$ qui avait commencé dès 1915. De nouvelles recettes de pain furent proposées dans la presse et on peut supposer qu'elles eurent un certain retentissement puisqu'elles sont mentionnées dans plusieurs journaux intimes. Par ailleurs, le petit-déjeuner se transforma. Les Schrippen, Brötchen, Semmel, Knüppel, ces petits pains ronds, toujours consommés aujourd'hui en Allemagne, furent interdits, alors qu'ils constituaient la base du petit-déjeuner. Le 11 août 1917, un article intitulé «Redonnez-nous notre Schrippe [petit pain] ! ne fut qu'un indice parmi d'autres de la frustration qui s'enracinait cette année-là ${ }^{(89)}$. Il pointait un problème auquel les citadins étaient confrontés depuis l'hiver 1916-1917. "Dans mon enfance, nous avions souvent faim. [...] La plupart du temps, nous allions à l'école sans notre pain de récré

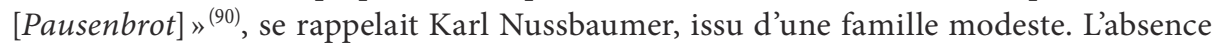
de ce goûter, souvent composé de deux tranches de pain et de beurre, symbolisait son expérience de la faim. Les ersatz ne firent quaggraver cette rupture par leur manque de diversité: pour Otto Schneider, le navet sorti des cartables était «réputé répugnant et pourtant indispensable», faisait «office d'ersatz au goûter [Pausenbrotersatz]» et était «grignoté par les enfants tels des lapins pour apaiser leur faim » ${ }^{(91)}$.

Cette perception gustative est confirmée dans un article de septembre 1917. Issu de la revue médicale allemande hebdomadaire, la Deutsche Medizinische Wochenschrift, son auteur y soulignait une nette dégradation de la qualité du pain, son goût et son odeur ayant "souvent été perçus comme répugnants et nauséabonds». Le pain de guerre (Kriegsbrot) y est rendu responsable de la "propagation actuelle des maladies intestinales " ${ }^{(92)}$. Parfois, cet aliment n'est pas nommé explicitement: la mère d'Otto Rees tomba gravement malade en 1917 et put alors rejoindre son fils qui avait été envoyé dans une famille d'accueil en Suisse ${ }^{(93)}$. Mais à la fin de son séjour, au cours de l'hiver, le temps se fit de nouveau très rude et la fête de Noël fut marquée par la prière, mais se déroula sans sapin et sans cadeaux: "En dépit du repos en Suisse, ma mère était très affaiblie et pleurait [...] ce fut le Noël le plus triste de ma jeunesse» ${ }^{\left({ }^{9}\right)}$.

Sur le plan sanitaire, ce sont les personnes vulnérables (personnes âgées, enfants et malades) qui furent alors les plus touchées. Les statistiques de l'hôpital de Homburg montrent que le pic de mortalité, s'élevant à 50,7 \% des patients, se situe en 1917. Toujours à Homburg, le tour de tête des nouveau-nés diminua de 29,4 \% entre 1916 et 1917 et de $25,6 \%$ entre 1917 et 1918 . Il se stabilisa entre 1918 et 1919 avec une diminution de 1,6\%, marquant une amélioration relative ${ }^{(95)}$. À la fin de l'année 1917, Hildegard

88 Ibid., p. 87, «Die deutsche Brotfrage in der Schule», 17 janv. 1915.

89 BArch R/8034/II 1451, Brot und Bäckererzeugnisse (note 5), vol. 4, p. 159, "Gebt uns die Schrippe wieder! », 11 août 1917.

90 TArch 1702, Journal intime de Karl Nußbaumer (1915-1986). Lebenserinnerungen, 1983, p. 4.

91 TArch 2162, Journal intime d'Otto Schneider (note 67), chap. «Heimatfront 1914-18», p. 4.

92 BArch R86/ 3281 (note 53), «Nährschäden der Kriegskost», Kölnische Volkszeitung, n 248, $1^{\text {er }}$ juillet 1920 ; R8034/ II 1451 (note 81), «Kriegsbrot und Darmkrankheiten ». Cette affirmation s'appuie sur un rapport de la confédération des meuniers allemands.

93 Voir TArch 2162, Journal intime d'Otto Schneider (note 67), chap. «Heimatfront 1914-18», p. 10.

94 TArch 921, Journal intime d'Otto Rees, Kein Engel - kein Held (note 61), p. 10.

95 Voir BArch R86/3283, Gesundheitliche Folgen der Kriegsernährung, lettre de Walter Koch, directeur de l'hôpital de Homburg, au ministre de l'Intérieur, Munich, 18 mars 1920. 
Lehmann, née en 1901 et résidant à Berlin, nota un moment rare d'amélioration de l'alimentation. Bien qu'issue d'un milieu aisé, elle s'exprimait en des termes similaires à ceux d'Otto Rees à propos du pain. Elle qualifia le repas du 3 octobre 1917, composé de pain beurré (Butterstullen) et de viande de méchoui, de moment "grandiose ${ }^{(96)}$. D’après les souvenirs d'après-guerre d'Otto Schneider, "l'augmentation des rations, aussi réduite soit-elle, fit rêver, surtout celles en beurre et en pain qui eurent un effet aujourd'hui inimaginable ${ }^{(97)}$.

La sensibilité pour la valeur du pain se trouve ainsi aiguisée pendant la guerre et apparaît tout particulièrement en 1917, aussi bien dans la presse que dans les journaux intimes ${ }^{(98)}$. Par ailleurs, en raison de la dégradation qualitative et de l'approvisionnement sur le marché noir, très courant en dépit des interdictions ${ }^{(99)}$, la quantification officielle des rations quotidiennes ne permet qu'une appréhension très fragmentaire des réalités. En revanche, une analyse d'expériences individuelles permet de préciser et d'approfondir l'étude, tout en atteignant une certaine représentativité grâce à la diversité géographique et sociale des acteurs choisis. Aussi ponctuelles et personnelles soient-elles, ces expériences mettent au jour des tendances évidentes au sein de réalités subjectives multiples.

C'est à travers la description des mœurs, des pratiques, des espoirs et des déceptions, mais aussi des corps, que se dessine un tournant sous-estimé et pourtant majeur en 1917. Les perceptions de l'alimentation en général, très souvent représentées par le pain, se trouvent alors altérées. Le pain devient lui-même, pour de nombreux acteurs politiques, militaires ou civils, une denrée indispensable à la survie, mais aussi à la victoire. Sa dimension symbolique est de plus en plus souvent mentionnée et son rôle nutritionnel s'accroît. La peur du manque de pain constitue alors l'un des motifs principaux au sein des écrits de l'intime. Les attentes se calquent sur l'évolution des rations de pain, mais aussi sur celle des matières grasses telles que le beurre. La fréquence avec laquelle la presse informait des augmentations et des diminutions des rations de pain confirme l'hypothèse. Nous observons une transposition subjective d'une signification symbolique sur un objet dont l'importance nutritionnelle se trouve surévaluée. Les sources concernant le pain dépassent bien souvent l'aliment lui-même: cette évolution s'opère dès l'année 1916 et se poursuit bien après 1917, mais l'année 1917 apparaît bien comme l'année charnière de cette évolution.

\section{Résumé}

L'article défend la thèse d'après laquelle les usages et témoignages concernant le pain de guerre, une sinon la denrée principale par excellence, dévoilent des ruptures à plusieurs niveaux en 1917. D'abord, celles-ci sont politiques et administratives. Ensuite, elles

96 TArch 71, Journal intime d'Hildegard Lehmann (1901-1964), p. 5.

97 TArch 2162, Journal intime d'Otto Schneider (note 67), chap. « Heimatfront 1914-18».

98 Ibid., p. 4. Dans ses mémoires, Otto Schneider consacre un chapitre au "pain de tous les jours». La dégustation de pain et de beurre a aussi marqué Margarethe Schützinger (TArch. 877 / I.1, Journal intime de Margarethe Schützinger, rédigé de 1885 à 1947).

Voir L. Lippmann, Mein Leben und meine amtliche Tätigkeit (note 58). 
sont aussi perçues par une dégradation quantitative et gustative. En retraçant chronologiquement les évolutions du rapport des politiques, de la presse et des acteurs sociaux à cet aliment, les traces de souffrances dues aux carences alimentaires se densifient clairement en cette année charnière de la guerre. C'est un moment de bouleversements profonds, non seulement des structures politiques et sociales, mais aussi du quotidien des individus. L'article propose une lecture différente d'une année de guerre décisive en interrogeant les conséquences d'attentes déçues, influencées par les mours culinaires, profondément incarnées dans les papilles et les esprits d'une population urbaine à bout de force.

\title{
Zusammenfassung
}

Der Artikel verteidigt die These, dass die Bräuche und Zeugnisse in Bezug auf das Kriegsbrot - ein, wenn nicht das Hauptnahrungsmittel schlechthin - es ermöglichen, Zäsuren auf verschiedenen Ebenen im Jahr 1917 aufzudecken. Zuerst sind diese auf der politischen und der Verwaltungsebene anzusiedeln. Außerdem werden sie auch durch die abnehmende Brotqualität und Brotmenge wahrgenommen. Indem chronologisch nachvollzogen wird, wie Politiker, Presse und soziale Akteure zum Kriegsbrot stehen, verdichten sich in diesem Wendejahr des Krieges deutlich die Anzeichen eines mit den Nahrungsmangeln in Verbindung stehenden Leids. Nicht nur im Hinblick auf politische und soziale Strukturen, sondern auch in Bezug auf den Alltag der Individuen ist es ein Jahr des Umbruchs. Der Artikel bietet eine andere Lesart eines entscheidenden Kriegsjahres, indem er nach den Konsequenzen enttäuschter Erwartungen fragt. Sie werden von kulinarischen Bräuchen beeinflusst und sind tief in den Körpern und den Gemütern einer erschöpften städtischen Bevölkerung verankert.

\begin{abstract}
The article argues that the habits and contemporary eyewitness reports regarding war bread - one, if not the main dish overall - enables to discover breaks on different levels during the year 1917. To begin with, these breaks happened on the political and the administrative level. Furthermore, they were accompanied by a decreasing bread quality. By analyzing the historical sources in chronological order, one can understand how politicians, the press and social actors were thinking about bread. Evidence of pain due to the food situation leads to the conclusion that this period was a real turning point, not only on the political and the social level, but also regarding the everyday life of individuals. The article shows a new way to interpret an important year of the war by looking at the consequences of the disappointed hopes which had been influenced by culinary rituals and were very deeply rooted in the bodies and spirits of an exhausted urban population.
\end{abstract}

